\title{
Printed Spiral Winding Inductor With Wide Frequency Bandwidth
}

\author{
Chi Kwan Lee, Member, IEEE, Y. P. Su, Student Member, IEEE, and S. Y. Ron Hui, Fellow, IEEE
}

\begin{abstract}
Winding parasitic capacitance is a major factor limiting the bandwidth of an inductor. In this paper, 1) the traditional, 2 ) the alternating, and 3 ) the partial alternating winding methods are evaluated for the multilayer printed spiral winding inductors for megahertz operations. The self-capacitances of various winding structures are estimated by the summation of parasitic capacitance among the turns of a winding. The electric field energy distributions in the inductors are derived from the voltage profiles to illustrate the relative magnitudes of winding parasitic capacitances. The results show that parasitic capacitance reduction can be achieved by reducing stored electric field energy. The partial alternating winding method is found to have the widest frequency bandwidth with reduced number of through-hole vias for multilayer printed spiral winding design. The theoretical analysis has been confirmed with practical measurements. The results provide useful information for the optimal design of coreless or core-based high-frequency planar magnetics.
\end{abstract}

Index Terms-Planar magnetic device, printed circuit board inductors, spiral winding.

\section{INTRODUCTION}

$\mathbf{L}$ OW PROFILE and high compactness are attractive features for power converters in applications such as planar LCD monitors, laptop computers, and iPads in which slim product designs and light weight are critical. In general, the size of passive components of a converter is reduced as frequency increases. Present switching converters typically operate at the frequency range of 100-500 kHz. Megahertz operation (e.g., up to $10 \mathrm{MHz}$ and beyond) can further reduce the size of the transformers and inductors [1]-[4]. However, the parasitic capacitance of the inductor winding is a limiting factor for bandwidth of the switching operation. Together with the equivalent series resistance (ESR), it deteriorates the quality of inductor

Manuscript received November 11, 2009; revised July 26, 2010; accepted August 23, 2010. Date of current version October 5, 2011. This work was supported by the Research Grant Council of Hong Kong under Grant CityU 114708. Recommended for publication by Associate Editor B. Ferreira.

C. K. Lee is with the Department of Electrical Engineering, The Hong Kong Polytechnic University, Kowloon, Hong Kong (e-mail: eecklee@polyu.edu.hk).

Y. P. Su is with the Center for Power Electronics Systems, Virginia Polytechnic Institute and State University, Blacksburg, VA 24060 USA (e-mail: yipengsu@vt.edu).

S. Y. R. Hui is with the Centre for Power Electronics, City University of Hong Kong, Kowloon, Hong Kong and also with the Imperial College of Science and Technology, London, U.K. (e-mail: eeronhui@ cityu.edu.hk).

Color versions of one or more of the figures in this paper are available online at http://ieeexplore.ieee.org.

Digital Object Identifier 10.1109/TPEL.2010.2076318 at high frequency. In addition, this parasitic capacitance provides an undesired conducting path to the high-frequency signals and noise and therefore reduces the effectiveness of electromagnetic compatibility filters. Thus, any practical inductor design suitable for megahertz should have very small parasitic capacitance.

The development of planar magnetics has sparked off a new research direction in developing high power density and very low profile power converters [5]-[10]. With the use of printed circuit board (PCB) windings, the cost of the traditional manual winding process can be eliminated. Unlike traditional corebased magnetic components that have a limited selection of wire sizes and core shapes, the printed windings offer the flexibility of winding geometry, shape, size, and even individual turns configuration. The advantages of the coreless planar transformers and inductors [5], [6] have been confirmed by researchers from Philips Research Center [29]. Multilayer coupled stacked windings have been introduced to maximize the inductance in a limited area [11], [12] for very low power solid state circuits. The relatively large intrawinding capacitance between two winding layers has been identified for such structures for limiting the self-resonant frequency (SRF) of the device and therefore the operating frequency bandwidth.

For a given PCB winding, the parasitic capacitance depends on the dielectric constant, thickness of the dielectric laminate, and voltage distribution over the winding. Methods for examining the parasitic capacitance through the electric field energy distribution along the winding have been addressed in [13]-[16]. The effects of parasitic capacitance through reorganizing the turn-to-turn configurations can be found in [26]. In order to reduce the parasitic capacitance, an alternating winding method has been introduced in high frequency planar magnetic design [17], [18] to partially cancel the electric field strength between alternating turns. However, alternating all the windings through the PCB substrate requires special treatment on the PCB such as many via holes for linking individual turns in series. Such winding structure increases both winding resistance and the manufacturing cost.

This study investigates the feasibility of the new partial alternating winding method that combines the traditional and alternating winding arrangements for a multilayer configuration with reduced number of connection layers and via holes. The averaged voltage profile and stored electrical field energy on each layer are evaluated. A systematic way to find the optimal number of alternating turns to obtain the widest operating frequency bandwidth is included. Finally, the influence of the nearby ferromagnetic components such as electromagnetic (EM) shields is discussed. 


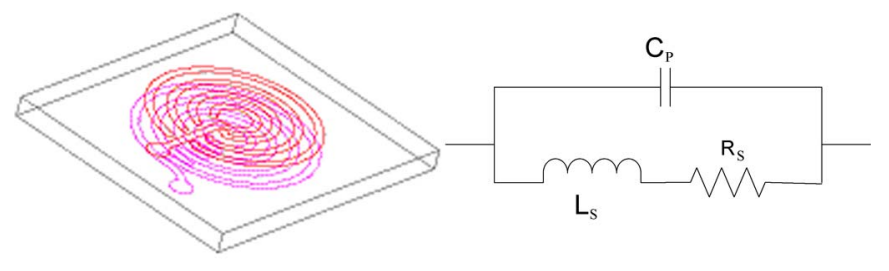

(a)

(b)

Fig. 1. 3-D view and equivalent lumped circuit model of a printed spiral winding inductor. (a) 3-D view of a printed spiral winding inductor. (b) Highfrequency small inductor lumped circuit model.

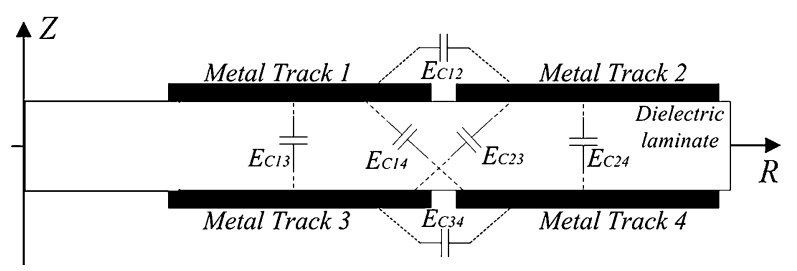

Fig. 2. Cross-sectional view of the winding in the half $R-Z$ plane showing the coupling paths of four metallic tracks between a dielectric laminate without electromagnetic shields.

\section{SElF-CAPACITANCE OF PRINTED SPIRAL WINDING INDUCTOR}

Fig. 1 shows a 3-D view of the physical structure and the equivalent lumped circuit model of a double-layer printed spiral winding inductor. When a voltage is applied across the inductor, the voltage gradient in the winding causes an electric field, which in turn creates a capacitive effect between two adjacent conductors. Such capacitive coupling leads to increased selfcapacitance of the inductor, which has adverse effect in high frequency operation. Therefore, a small high-frequency inductor can be modeled as an impedance network as shown in Fig. 1(b). The SRF of the inductor is

$$
\mathrm{SRF}=\frac{1}{2 \pi \sqrt{L_{S} C_{P}}}
$$

where $L_{S}$ and $C_{P}$ are the inductance and self-capacitance of the inductor, respectively. $R_{S}$ represents the resistance of the winding. The calculation for the required radius and the number of turns for a given inductance of the winding can be found in [22] and [23].

The self-capacitance of an inductor is equal to the summation of parasitic capacitance between the turns of a winding and their surrounding materials (e.g., circuit board, core, and EM shields). Fig. 2 shows the coupling paths of four metallic tracks between a dielectric laminate of a 4-turn double-layer printed spiral winding inductor without EM shields.

Assume that the potential distribution is proportional to the lengths of the metallic tracks. The electrical energy $E_{i j}$ associated with any two metallic tracks and the total electrical energy $E_{\text {total }}$ associated with this structure can be expressed by (2) and (3), respectively. The variable $V_{A}$ is the beginning potential difference between two metallic tracks, $V_{B}$ is the ending potential difference, $C_{i j}$ is the low frequency per unit length capacitance between the two metal surfaces and $l$ is the length of the two

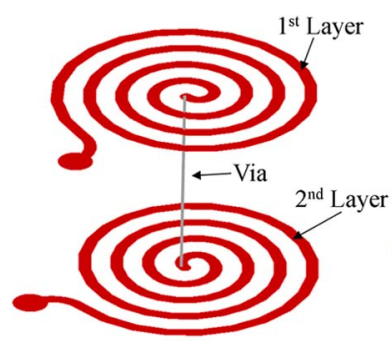

(a)

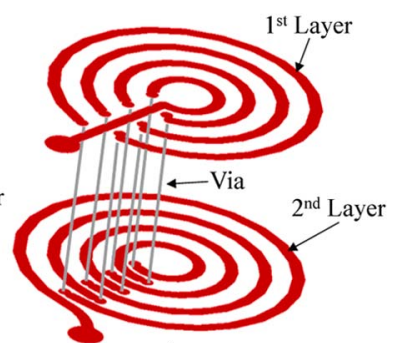

(b)
Fig. 3. (a) Traditional winding method and (b) alternating winding method for the double-layer printed spiral winding inductor.

metallic tracks. The detailed derivation of (2) is provided in the Appendix.

$$
\begin{aligned}
E_{i j} & =\frac{1}{6} C_{i j} l_{i j}\left[V_{A . i j}^{2}+V_{A, i j} V_{B, i j}+V_{B, i j}^{2}\right] \\
E_{\text {total }} & =\frac{1}{2} \sum_{i=1}^{N} \sum_{j=1}^{N} E_{i j} .
\end{aligned}
$$

The low frequency per unit length capacitance $C_{i j}$ can be precisely predicted either by finite element analysis simulation [19] or by theoretical static electric field calculation [20], [21]. Generally, the metallic track width is much larger than the spacing and thickness of the dielectric laminate in the planar magnetic design. Thus, the associated electrical energy between the tracks on the opposite sides of the dielectric laminate are the most significant terms in (3), i.e., $E_{13}$ and $E_{24}$ in Fig. 2. The selfcapacitance of the inductor is

$$
C_{S}=\frac{2 E_{\text {total }}}{V_{L}^{2}}
$$

where $V_{L}$ is the voltage applied to the inductor.

\section{DOUble-LAYER PRINTED SPIRAL WINDING INDUCTOR}

\section{A. Traditional and Alternating Winding Methods}

Fig. 3(a) and (b) illustrates the traditional and alternating winding methods for a double-layer printed spiral winding inductor. The traditional winding method starts the winding from the outermost loop of the first layer. After completing the innermost loop on the first layer, it crosses the dielectric laminate to the second layer through a via hole. Then, the second layer winding will spiral from the centre to the outermost loop. Due to the relatively large potential difference and long track length on the outer loops, the parasitic capacitance of this winding method is high and the SRF is low.

The alternating winding method is similar to the multisection winding technique that has been adopted in the traditional bobbin-type core-based inductors or transformers. The objective is to reduce the potential difference of the two adjacent conductors resulting in low parasitic capacitance. For a doublelayer alternating spiral winding inductor design, the winding alternates between the dielectric laminate for each successive loop. Each alternating cycle can be considered as one section. Therefore, a single winding with $N$ turns can be split into $(N / 2)$ 


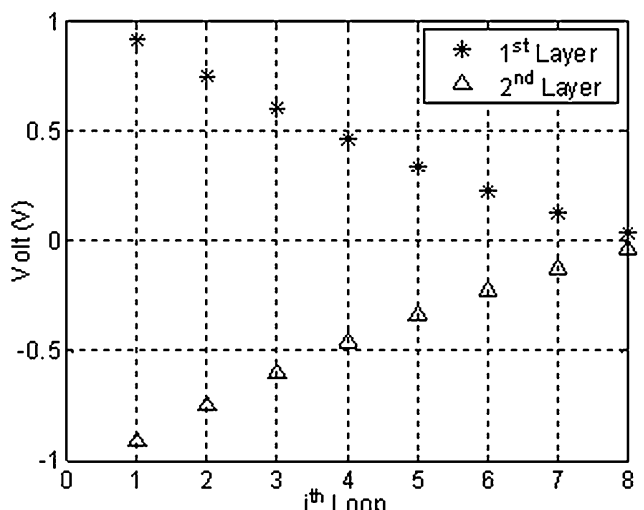

(a)

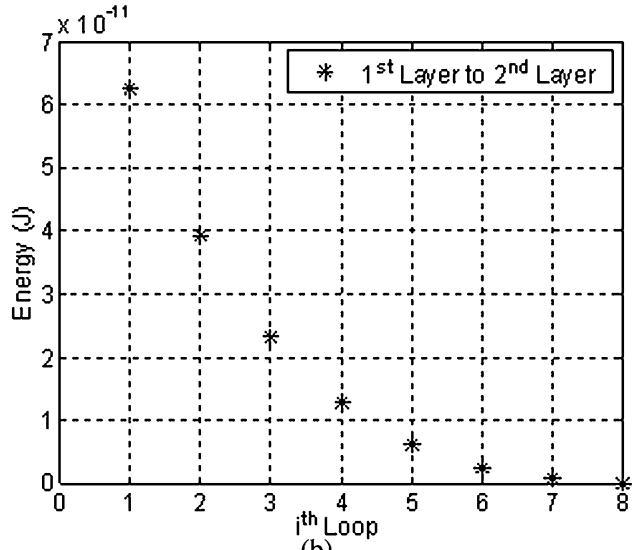

(b)

Fig. 4. (a) Averaged voltage profile and (b) energy profile of the double-layer 16-turn printed spiral winding inductor using the traditional winding method.

sections in a double-layer configuration. In this way, the number of turns and the footprint area are the same as the traditional winding method. Therefore, it is expected that their inductance values should be similar (see, e.g., [17], [18]).

\section{B. Voltage and Electrical Energy Profiles}

To illustrate the capacitive coupling effect of the two different winding methods, two 16-turn double-layer printed spiral winding inductors have been selected. The dimensional parameters are reported in Section V. Assume that the inductors are connected to $\pm 1 \mathrm{~V}$. The voltage gradient along the winding can be calculated by a linear drop of voltage with length of metal track. The voltage profiles of the two winding methods are shown in Figs. 4(a) and 5(b), respectively. Their associated electrical energy between the tracks on the opposite sides of the dielectric laminate can be plotted as shown in Figs. 4(b) and 5(b), respectively. For each layer, we label the outermost loop as the first loop and the innermost as the eighth loop. It is important to note that the electrical potential difference between the tracks on the opposite sides of the dielectric laminate in the alternating winding method [see Fig. 5(a)] is much smaller than that in the traditional winding method [see Fig. 4(a)]. In Fig. 5(b), the associated electrical energy between the dielectric laminate (first layer to second layer) has been reduced substantially.

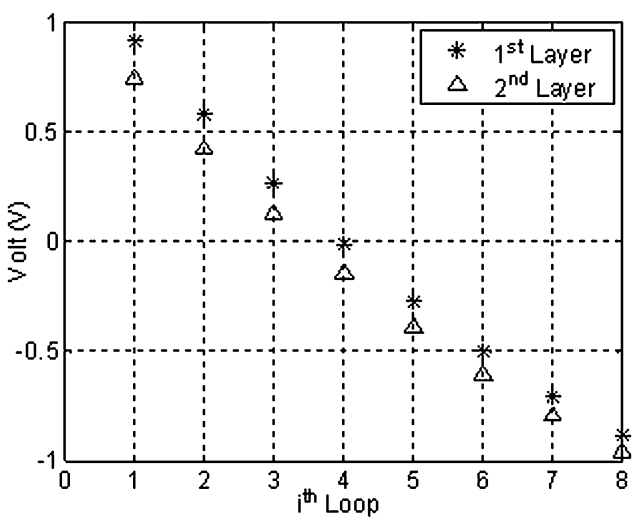

(a)

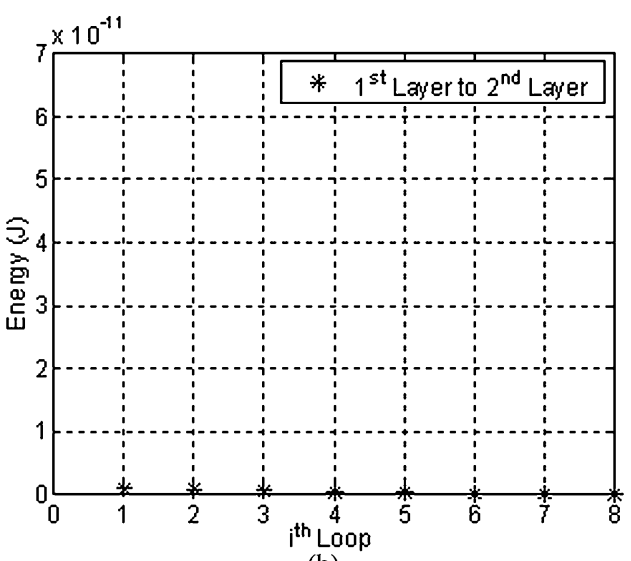

(b)

Fig. 5. (a) Averaged voltage profile and (b) energy profile of the double-layer 16-turn printed spiral winding inductor using the alternating winding method.

\section{MUltilayer PRINTEd SPIRAL Winding INDUCTOR}

\section{A. Traditional, Alternating, and Partial Alternating Winding Methods}

Fig. 6(a)-(c) illustrates three winding methods for a fourlayer printed spiral winding inductor namely the traditional, alternating, and partial alternating methods. Multilayer configuration can be considered with several double-layer PCBs being stacked and connected together. The detailed winding arrangements and turns sequence of three winding methods are shown in Fig. 7. The numerical index on the conductors denotes the winding sequence. $N$ is the total number of turns of the winding. The proposed partial alternating winding method divides the winding into two sections: 1) the alternating section and 2 ) the traditional section. The outer loops of the spiral are alternating to reduce the parasitic capacitance. The inner loops that have a relatively small effect on the parasitic capacitance will be wound in a traditional manner to balance the potential difference on each dielectric laminate. As the outer loops have the longer track length than inner loops, the interconnection between the second and the third layer can be connected at outermost loops to minimize their potential difference. $M$ is the number of turns in the alternating section of the partial alternating winding method. 


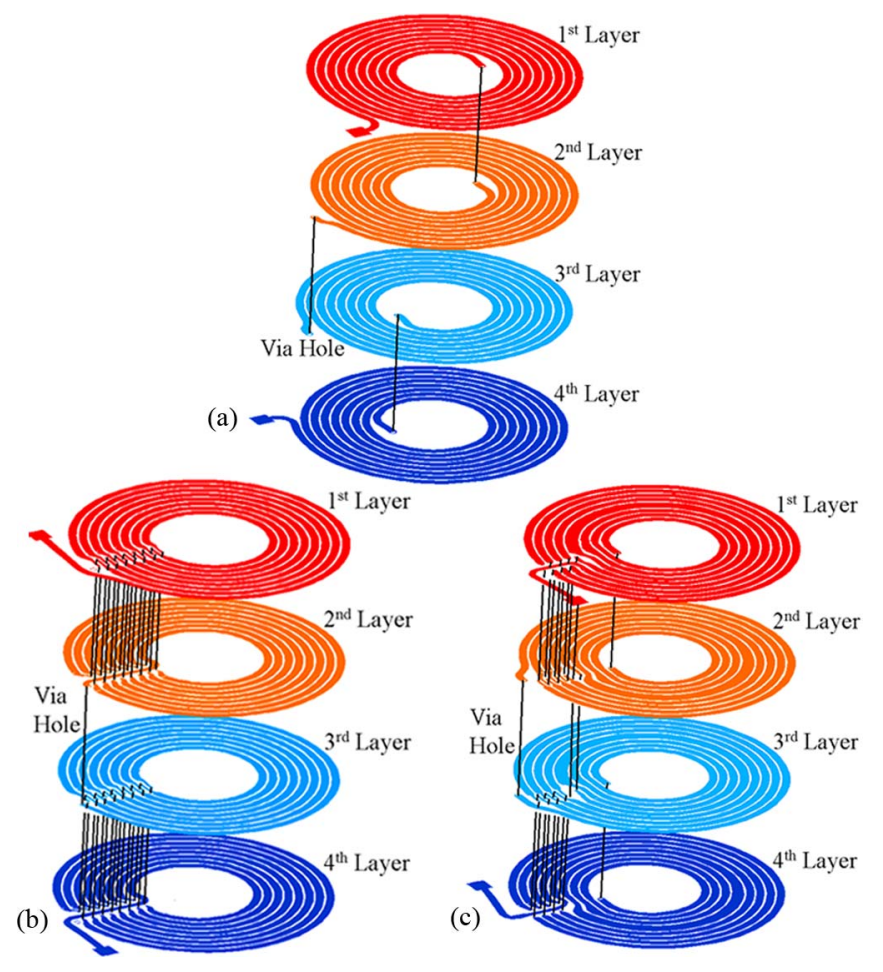

Fig. 6. Four-layer printed spiral winding inductor: (a) traditional winding method, (b) alternating winding method, and (c) partial alternating winding method.

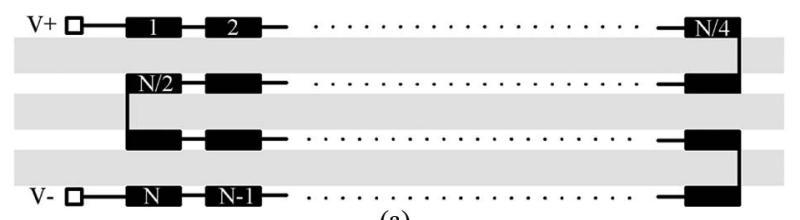

(a)

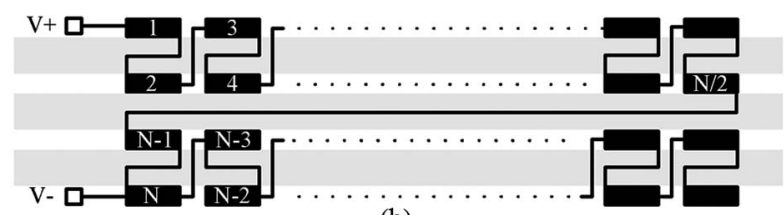

(b)

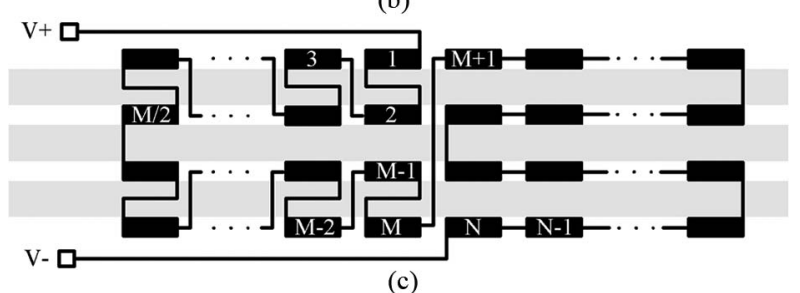

(c)

Fig. 7. Detailed winding arrangement of a four-layer printed spiral winding inductor: (a) traditional winding method, (b) alternating winding method, and (c) partial alternating winding method.

\section{B. Voltage and Electrical Energy Profiles}

A 32-turn four-layer printed spiral winding inductor has been selected. The voltage profile and the associated electrical energy profiles of the three winding methods can be plotted as shown in Figs. 8-10. A comparison between Fig. 8(b) and Fig. 9(b) shows that the associated electrical energy in the first layer

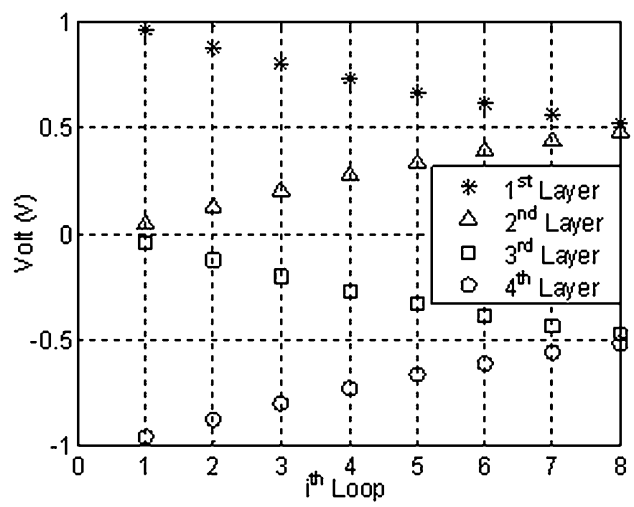

(a)

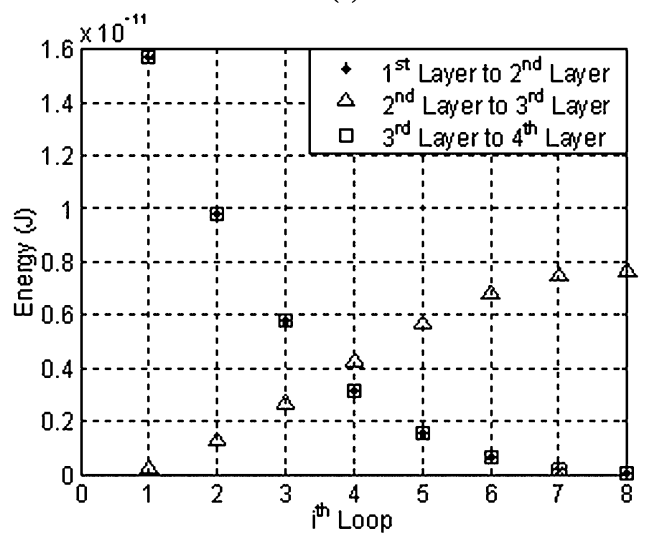

(b)

Fig. 8. (a) Averaged voltage profile and (b) energy profile of a four-layer 32-turn printed spiral winding inductor using the traditional winding method.

to the second layer and the third layer to the fourth layer has been reduced by the alternating winding method. However, it is important to note that the energy value has been increased in the second layer to the third layer when compared with the traditional winding method. This is due to the potential divider effect of the reduced potential difference in the first layer to the second layer and the third layer to the fourth layer as shown in Figs. 8(a) and 9(a). In Fig. 10, the new partial alternating winding method shows a well balance of stored energy in layers and with reduced number of via holes.

\section{Optimal Number of Alternating Turns}

It is important to determine the optimal number of turns that have to be alternating, so that an improved component can be obtained by thoughtfully balancing the electrical energy. The first step in the optimization is to assume a value $M$ for the number of turns in the alternating section. $M$ should be a multiple of the number of layers. A good value to start with is the turns number close to that formed by half of total winding length. The next step is to generate the voltage profiles $V_{A, i j}$ and $V_{B, i j}$ of the metallic tracks according to the winding sequence and the length of individual loop. The summations of associated electrical energy in the alternating winding section $E_{\text {alt }}$, the traditional winding section $E_{\text {trad }}$, and the boundary section $E_{\text {bound }}$ are expressed by (5)-(7). A synthesis procedure can 


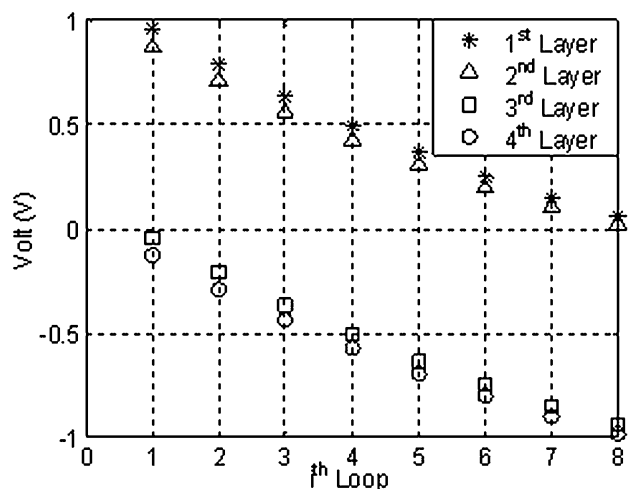

(a)

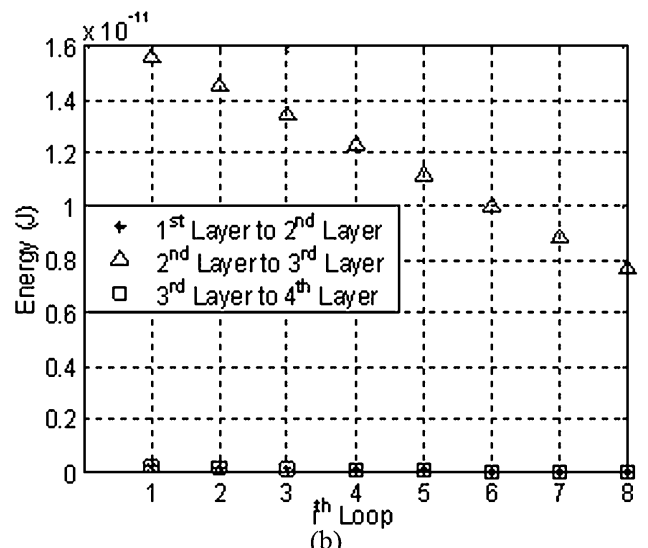

(b)

Fig. 9. (a) Averaged voltage profile and (b) energy profile of a four-layer 32-turn printed spiral winding inductor using the alternating winding method.

be implemented using MATLAB [27]. The associated electrical energy of different $M$ values can be calculated as

$$
\begin{aligned}
E_{\text {alt }} & =\frac{1}{2} \sum_{i=1}^{M} \sum_{j=1}^{M} E_{i j} \\
E_{\text {trad }} & =\frac{1}{2} \sum_{i=M}^{N} \sum_{j=M}^{N} E_{i j} \\
E_{\text {total }} & =E_{\text {alt }}+E_{\text {trad }}+E_{\text {bound }} .
\end{aligned}
$$

The selected 32-turn four-layer printed spiral winding inductor is used to illustrate the change of associated electrical energy with different numbers of $M$ value. The plot of the associated electrical energy of the alternating winding section $E_{\text {alt }}$, the traditional winding section $E_{\text {trad }}$, and the boundary section $E_{\text {bound }}$ for different number of $M$ are shown in Fig. 11. The plot of the total associated electrical energy for different numbers of $M$ is shown in Fig. 12. It can be seen that a good energy balance is achieved between the alternating winding section and the traditional winding section when $M$ is equal to 12 .

\section{PRACTICAL Verification}

Prototypes have been built to verify the characteristics of these three winding methods. The inductor has 8 turns on each layer and the dimensional parameters are outer radius $R_{\text {out }}=$ $21.4 \mathrm{~mm}$, dielectric laminate thickness $z=0.2 \mathrm{~mm}$, metal track

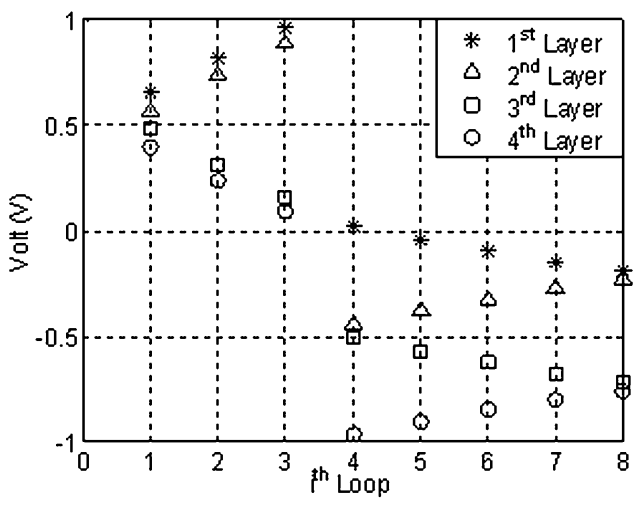

(a)

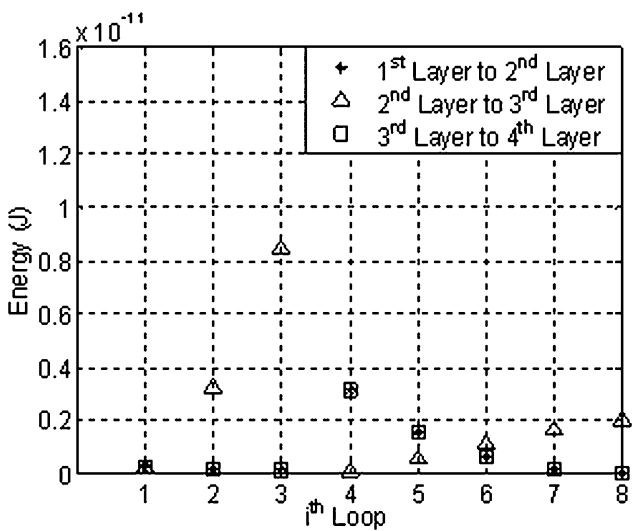

(b)

Fig. 10. (a) Averaged voltage profile and (b) energy profile of a four-layer 32-turn printed spiral winding inductor using the partial alternating winding method.

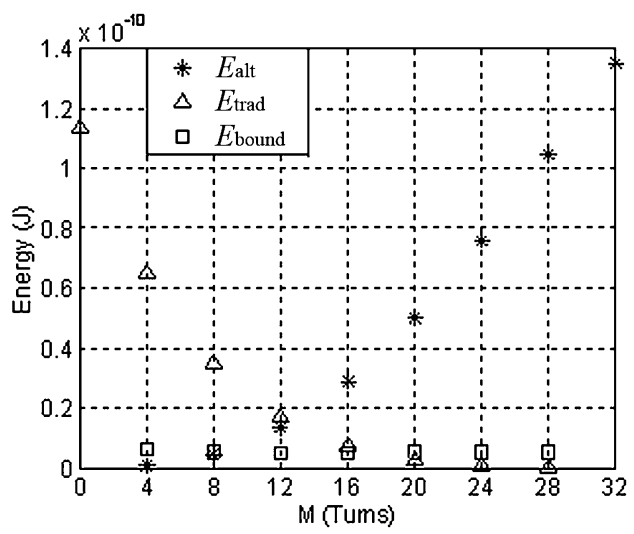

Fig. 11. Plot of the electrical energy $E_{\text {alt }}, \mathrm{E}_{\mathrm{trad}}$, and $E_{\mathrm{bound}}$ with different number of $M$.

width $w=1.3 \mathrm{~mm}$, thickness $h=70 \mu \mathrm{m}$, and clearance $s=$ $0.3 \mathrm{~mm}$. The PCB is made of FR-4 material with permittivity of 4.4. Fig. 13 illustrates the dimensional parameters in the half $R-Z$ plane. The central windings will be removed to reduce the eddy currents losses because they have a small contribution to the inductance but have adverse effects (increased resistance) on the inductor quality. The via hole diameter is $0.5 \mathrm{~mm}$ so that the conducting area is closed to the metallic track width. In order to keep the same footprint area of the inductors, track narrowing at the interconnection points is necessary for placing the via 


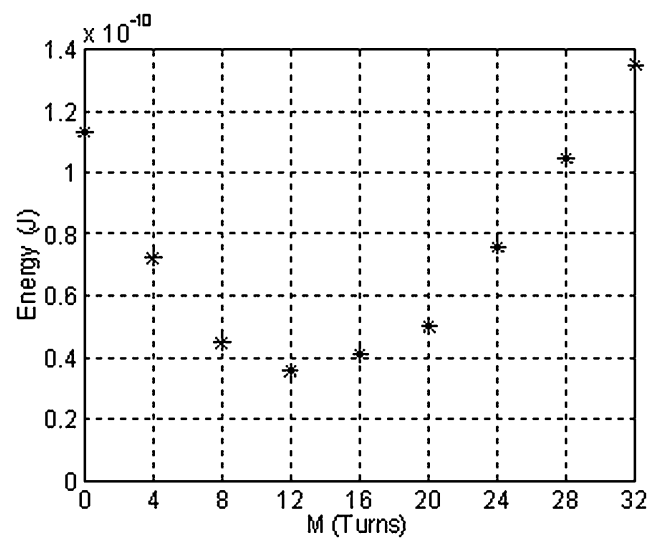

Fig. 12. Plot of the total associated electrical energy $E_{\text {total }}$ with different number of $M$.

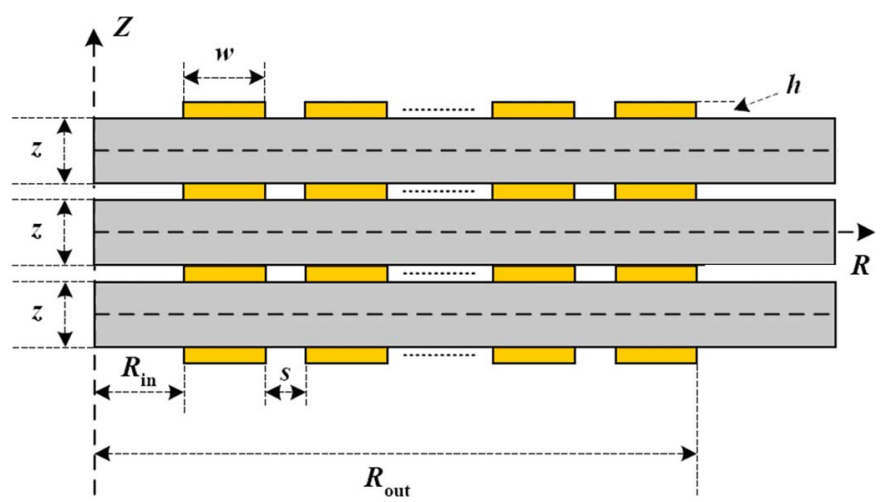

Fig. 13. Dimensional parameters of the printed spiral winding in the half $R-Z$ plane.

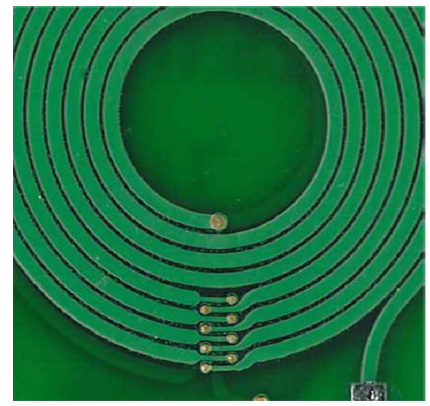

(a)

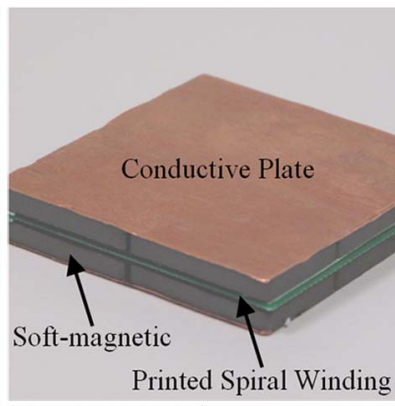

(b)
Fig. 14. (a) Photograph of the track narrowing at the interconnection points for placing the via hole in partial alternating winding methods. (b) Photograph of the printed spiral winding covered by EM shields.

hole in alternating and partial alternating winding methods [see Fig. 14(a)]. The calculated inductance and self-capacitance of the double-layer and four-layer printed spiral inductors with 8 turns on each layer are summarized in Table I. According to the calculation from Section IV-C, $M=12$ is chosen for the optimal number of alternating turns in the partial alternating winding method.

The impedance of the inductors is measured by Agilent 4294: A precision impedance analyzer. Data points are recorded from
TABLE I

CAlculated Inductance AND SElF-CAPACITANCE OF THE PRINTEd SPIRAL Winding INDUCTOR USING THE TRADITIONAL, ALTERNATING, AND PARTIAL ALTERNATING WINDING METHODS

\begin{tabular}{|c|c|c|c|}
\hline & $\mathrm{C}_{\mathrm{P}}$ & & \\
\hline \multicolumn{4}{|l|}{ Double-layer } \\
\hline Type of winding method & Traditional & Alternating & $\begin{array}{l}\text { Partial } \\
\text { Alternating }\end{array}$ \\
\hline $\begin{array}{l}\text { Calculated } \\
\text { inductance Ls (uH) }\end{array}$ & 8.63 & 8.63 & -- \\
\hline $\begin{array}{l}\text { Calculated self- } \\
\text { capacitance } \mathrm{Cp}(\mathrm{pF})\end{array}$ & 81.16 & 2.54 & -- \\
\hline $\begin{array}{l}\text { Calculated self-resonant } \\
\text { frequency }(\mathrm{MHz})\end{array}$ & 6.01 & 33.99 & -- \\
\hline \multicolumn{4}{|l|}{ Four-layer } \\
\hline $\begin{array}{l}\text { Calculated } \\
\text { inductance Ls (uH) }\end{array}$ & 33.69 & 33.69 & 33.69 \\
\hline $\begin{array}{l}\text { Calculated self- } \\
\text { capacitance } \mathrm{Cp}(\mathrm{pF})\end{array}$ & 56.72 & 49.57 & 17.88 \\
\hline $\begin{array}{l}\text { Calculated self-resonant } \\
\text { frequency SRF (MHz) }\end{array}$ & 3.64 & 3.89 & 6.48 \\
\hline
\end{tabular}

$40 \mathrm{~Hz}$ to $50 \mathrm{MHz}$. The inductance, self-capacitance and ESR are determined by the SRF and impedance curve [28].

\section{DISCUSSION}

\section{A. Impedance Characteristics}

The frequency responses of the impedance $|Z|$ and ESR of the double-layer printed spiral inductor prototypes from $10 \mathrm{kHz}$ to $50 \mathrm{MHz}$ are plotted in Fig. 15(a) and (b), respectively. The result shows that the inductor using the traditional winding method will resonate and lose its inductive effect at $5.53 \mathrm{MHz}$. The inductor using the alternating winding method can be operated up to $27.41 \mathrm{MHz}$. This result indicates that the parasitic capacitance in the winding is relatively small. Fig. 16(a) and (b) show the detailed frequency responses from $1 \mathrm{MHz}$ to $10 \mathrm{MHz}$. It is clear that the inductor using the alternating winding method has a wide frequency response. The impedance $|Z|$ appears as a straight line against the frequency. The measurement results also show that the ESR of the inductor using the alternating winding method is smaller than that using the traditional winding method. It is evident that the extra resistance due to via holes and track narrowing (for connecting the layers) is relatively small when compared with the ESR of the winding in high frequency.

The measured frequency responses of the four-layer printed spiral winding inductors using the three winding methods are shown in Fig. 17. It should be noted that the SRF of the inductor using the proposed partial alternating winding method is $6.58 \mathrm{MHz}$, whilst those of the traditional and alternating winding methods are only about $3.54 \mathrm{MHz}$ and $3.93 \mathrm{MHz}$, respectively. The detailed impedance and ESR from $1 \mathrm{MHz}$ to $3 \mathrm{MHz}$ are plotted in Fig. 18. The inductors using the traditional and 


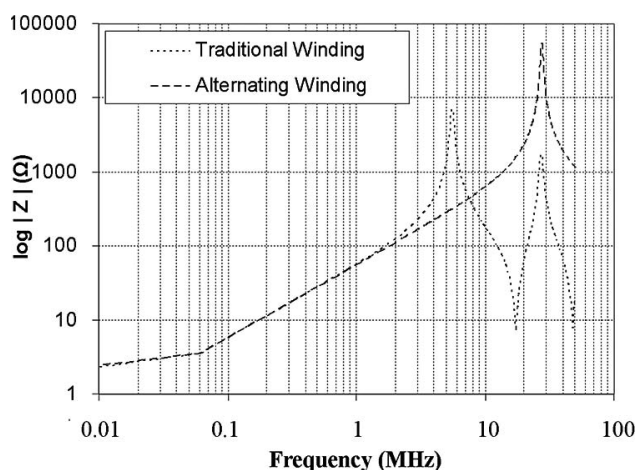

(a)

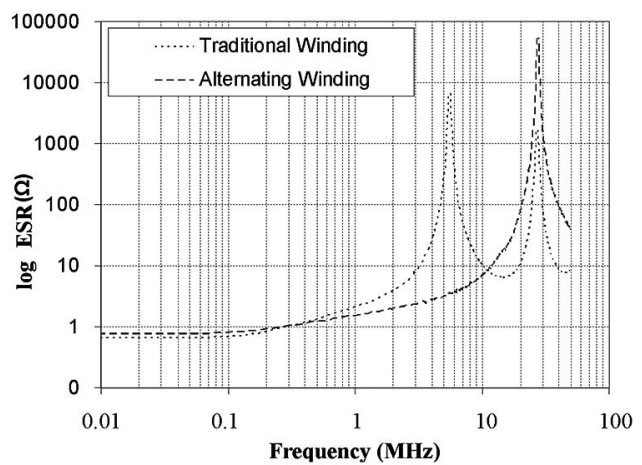

(b)

Fig. 15. Measured frequency response of the double-layer printed spiral inductor using the traditional and alternating winding methods. (a) Impedance from $10 \mathrm{kHz}$ to $50 \mathrm{MHz}$ in log scale and (b) equivalent series resistance.

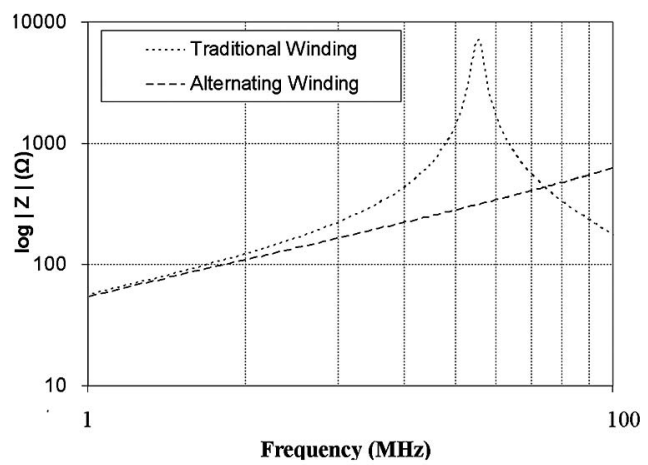

(a)

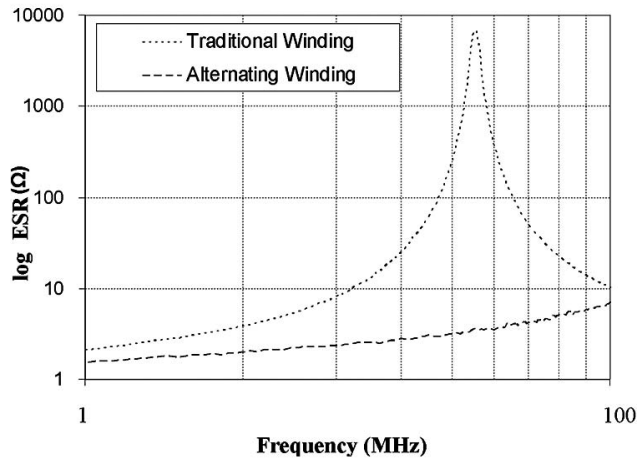

(b)

Fig. 16. Measured frequency response of the double-layer printed spiral inductor using the traditional and alternating winding methods. (a) Impedance from $1 \mathrm{MHz}$ to $10 \mathrm{MHz}$ in log scale and (b) equivalent series resistance.

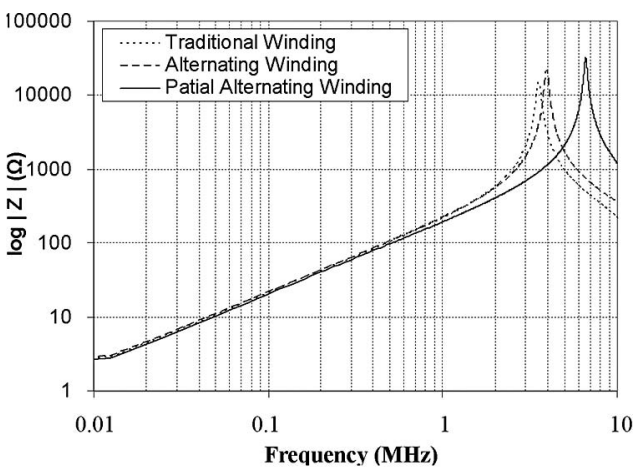

(a)

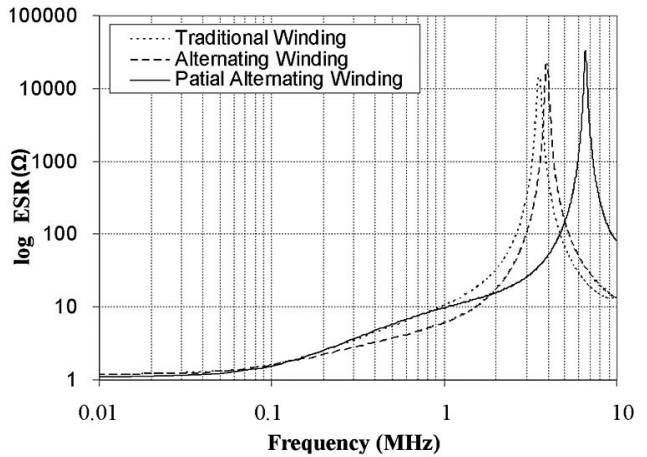

(b)

Fig. 17. Measured frequency response of the four-layer printed spiral inductor using the traditional, alternating, and partial alternating winding methods. (a) Impedance from $10 \mathrm{kHz}$ to $10 \mathrm{MHz}$ in log scale and (b) equivalent series resistance.

alternating winding methods only have linear impedances in the low frequency range less than $750 \mathrm{kHz}$. The use of the partial alternating winding method can improve the frequency response up to $2 \mathrm{MHz}$. Fig. 18(b) shows that the inductor using the alternating winding method has the lowest ESR at frequency less than $1 \mathrm{MHz}$. While the ESR of the traditional and partial alternating winding methods are similar in low frequency $(<1 \mathrm{MHz})$, a substantial improvement by using the partial alternating winding method can be observed when the frequency is beyond $2 \mathrm{MHz}$. The influence of the interconnections and the track width on the dc resistance of the spiral winding has been discussed in [23]. Therefore, further studies in the effect of the ESR, the ac winding resistance, losses and the $Q$-factor by different winding methods or reorganizing the turn-to-turn configurations for the printed spiral winding are needed. As a result, the design guideline for a low ESR and wide operating frequency planar inductor can be formulated. An optimal design approach for a given inductor size and inductance can be obtained.

\section{B. Equivalent Circuit Model}

Table II shows a comparison of the inductors using the equivalent circuit model. The calculated and measured parameters are in very good agreement. It is confirmed that both inductors have similar inductances and ESR values in the low-frequency range. The small variations in the inductance and ESR values come from the different geometrics layout, layer-to-layer 


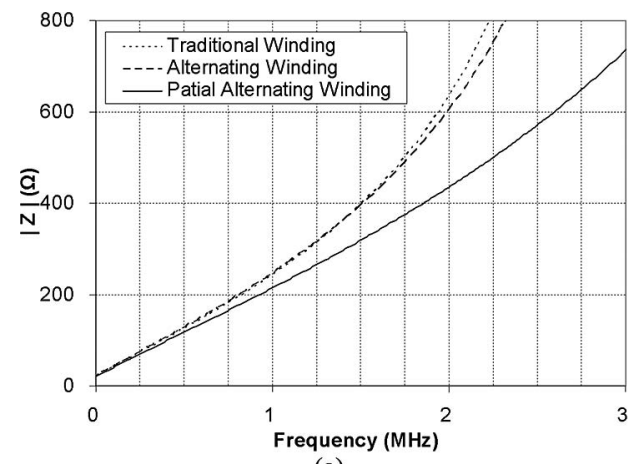

(a)

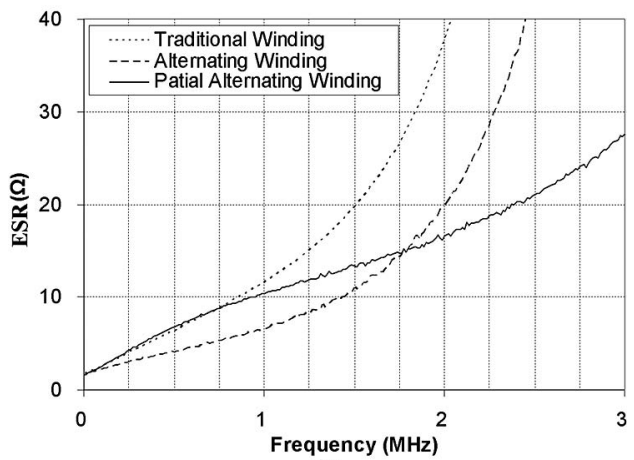

(b)

Fig. 18. Measured frequency response of the four-layer printed spiral inductor using the traditional, alternating, and partial alternating winding methods. (a) Impedance from $1 \mathrm{MHz}$ to $3 \mathrm{MHz}$. (b) Equivalent series resistance.

interconnections and PCB manufacturing process used. The self-capacitance of the double-layer printed spiral inductor using alternating winding methods is greatly reduced from $94.13 \mathrm{pF}$ to $3.90 \mathrm{pF}$. The proposed partial alternating winding method is found to be more effective in reducing the self-capacitance than the other two types of winding methods in multilayer configuration. Thus, the bandwidth of the inductor using the partial alternating winding method has an improvement of 1.8 times better than that using the traditional winding method. Elementary phasor circuit analysis of the inductor equivalent circuit model shows that the measured impedance can be expressed by (8).

$$
\begin{aligned}
Z= & \frac{R_{S}}{\left(1-\omega^{2} L_{S} C_{P}\right)^{2}+\omega^{2} C_{P}^{2} R_{S}^{2}} \\
& +j \frac{\omega L_{P}\left(1-\omega^{2} L_{S} C_{P}-\left(C_{P} R_{S}^{2} / L_{S}\right)\right)}{\left(1-\omega^{2} L_{S} C_{P}\right)^{2}+\omega^{2} C_{P}^{2} R_{S}^{2}} .
\end{aligned}
$$

The ESR is the real part of the measured impedance. The measured ESR is equal to the winding resistance when the inductor has a low self-capacitance $C_{P}$ value. The reduction of the parasitic capacitance in the winding not only increases the operating frequency bandwidth of the inductor, but also reduces the ESR of the inductor. Thus, the quality of the inductor at high frequency has been improved.
TABLE II

COMPARISON OF THE TRADITIONAL, ALTERNATING, AND PARTIAL

\begin{tabular}{|c|c|c|c|}
\hline \multicolumn{4}{|c|}{ Readings in ( ) are the measurements with EM Shields } \\
\hline \multicolumn{4}{|c|}{ Double-layer } \\
\hline Type of winding method & Traditional & Alternating & $\begin{array}{l}\text { Partial } \\
\text { Alternating }\end{array}$ \\
\hline Inductance Ls (uH) & $\begin{array}{l}8.80 \\
(47.09)\end{array}$ & $\begin{array}{l}8.66 \\
(41.68)\end{array}$ & -- \\
\hline Self-capacitance $\mathrm{Cp}(\mathrm{pF})$ & $\begin{array}{l}94.13 \\
(90.53)\end{array}$ & $\begin{array}{l}3.90 \\
(5.45)\end{array}$ & -- \\
\hline $\begin{array}{l}\text { Equivalent series } \\
\text { resistance } \operatorname{ESR}(\Omega) \\
\text { (a) } 40 \mathrm{~Hz} \\
\text { (a) } 1 \mathrm{MHz}\end{array}$ & $\begin{array}{l}0.61 \\
(0.65) \\
2.11 \\
(34.67)\end{array}$ & $\begin{array}{l}0.74 \\
(0.74) \\
1.51 \\
(13.48)\end{array}$ & -- \\
\hline $\begin{array}{l}\text { Measured self-resonant } \\
\text { frequency SRF }(\mathrm{MHz})\end{array}$ & $\begin{array}{l}5.53 \\
(2.44)\end{array}$ & $\begin{array}{l}27.41 \\
(10.56)\end{array}$ & -- \\
\hline \multicolumn{4}{|l|}{ Four-layer } \\
\hline Inductance Ls (uH) & $\begin{array}{l}33.1 \\
(183)\end{array}$ & $\begin{array}{l}33.7 \\
(181)\end{array}$ & $\begin{array}{l}30.9 \\
(156)\end{array}$ \\
\hline Self-capacitance $\mathrm{Cp}(\mathrm{pF})$ & $\begin{array}{l}61.6 \\
(60.6)\end{array}$ & $\begin{array}{l}48.7 \\
(49)\end{array}$ & $\begin{array}{l}18.9 \\
(21.2)\end{array}$ \\
\hline $\begin{array}{l}\text { Equivalent series } \\
\text { resistance } \operatorname{ESR}(\Omega) \\
@ 40 \mathrm{~Hz} \\
\text { @ } 1 \mathrm{MHz}\end{array}$ & $\begin{array}{l}1.16 \\
(1.19) \\
10.42 \\
(343.15)\end{array}$ & $\begin{array}{l}1.16 \\
(1.13) \\
5.99 \\
(152.92)\end{array}$ & $\begin{array}{l}1.03 \\
(1.07) \\
9.78 \\
(112.84)\end{array}$ \\
\hline $\begin{array}{l}\text { Measured self-resonant } \\
\text { frequency SRF (MHz) }\end{array}$ & $\begin{array}{l}3.54 \\
(1.51)\end{array}$ & $\begin{array}{l}3.93 \\
(1.69)\end{array}$ & $\begin{array}{l}6.58 \\
(2.77)\end{array}$ \\
\hline
\end{tabular}
ALternating Winding Methods For PRINTED SPIRAL Winding INDUCTORS

\section{Inductor With Nearby Ferromagnetic Components}

Measurements have been carried out on the inductors with EM shields to evaluate the coupling effect between the windings and nearby shielding materials. The impedance and ESR of the inductors are recorded. Two pieces of double-layer EM shields consisting of 3F4 soft-magnetic and conductive plates have been used for the experiments [24]. The distances of the printed spiral winding from the top and bottom EM shields are both $0.4 \mathrm{~mm}$. Table II shows that the inductance has been increased by the magnetic substrate. Consequently, the SRF is reduced. The high ESR value is due to the increased inductance and ac resistance in the winding. The nonuniform current distribution in the conductors due to skin and proximity effects have been discussed in [23]-[25]. It is important to note that the self-capacitance with and without EM shields are similar (the difference is less than $3 \mathrm{pF}$ ). This indicates that the electric coupling effect between the winding and EM shields is negligible. Thus, the frequency response of a printed spiral winding inductor is mainly relied on the winding configurations. The reduction of the self-capacitance of the inductor to improve the frequency bandwidth and reduce ESR in the winding for a printed spiral winding inductor is confirmed again. 


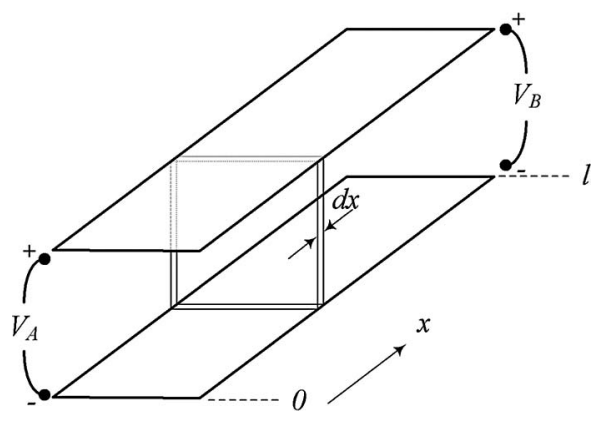

Fig. 19. Representation of two metal surfaces for winding self-capacitance calculation.

\section{CONCLUSION}

In this paper, three winding methods for the multilayer printed spiral winding inductors have been studied. In double-layer design, the alternating winding method greatly reduces (over 20 times in the example) the self-capacitance of the inductor The operating frequency bandwidth and the $Q$-factor (in the megahertz frequency range) of the inductor are substantially improved. In the multilayer design, the proposed partial alternating winding method combines the advantageous features of the traditional and alternating winding methods. The self-capacitance of the inductor can be reduced by alternating some of the outer loops metallic tracks. This method can be used to balance the electric field energy distributions in the winding. As a result, an optimal device can be obtained. The general procedure for the optimal number of alternating loops has been described. The frequency responses of three winding methods have been validated by using double-layer 16-turn and four-layer 32-turn devices. The proposed idea can be applied to planar inductors or transformers for high-frequency operations.

\section{APPENDIX}

The following procedure provides the derivation of (1). The detail derivation of self-capacitance due to winding layers and various arrangements is described in [26].

Consider Fig. 19; assuming a linear potential distribution of two surfaces, the potential difference $\Delta V$ at the element $d x$ is

$$
\Delta V=V_{A}+\left(V_{B}-V_{A}\right) \frac{x}{l}
$$

where $V_{A}$ is the potential difference between two metal surfaces at $x=0, V_{B}$ is the potential difference at $x=l, l$ is the length of the two metal surfaces. The associated electrical energy is

$$
d W=\frac{1}{2} C\left[V_{A}+\left(V_{B}-V_{A}\right) \frac{x}{l}\right]^{2} d x
$$

where $C$ is the low frequency per unit length capacitance between the two metal surfaces.

Integrating over the surface, total energy is

$$
W=\frac{1}{6} C l\left[V_{A}^{2}+V_{A} V_{B}+V_{B}^{2}\right] .
$$

\section{REFERENCES}

[1] J. S. Glaser, J. Nasadoski, and R. Heinrich, "A $900 \mathrm{~W}, 300 \mathrm{~V}$ to $50 \mathrm{~V}$ dc-dc power converter with a $30 \mathrm{MHz}$ switching frequency," in Proc. 24th Annu. IEEE Appl. Power Electron. Conf. Expo., Feb. 15-19, 2009, pp. $1121-1128$

[2] J. R. Warren, K. A. Rosowski, and D. J. Perreault, "Transistor selection and design of a VHF dc-dc power converter," IEEE Trans. Power Electron., vol. 23, no. 1, pp. 27-37, Jan. 2008.

[3] J. M. Rivas, Y. Han, O. Leitermann, A. D. Sagneri, and D. J. Perreault, "A high-frequency resonant inverter topology with low-voltage stress," IEEE Trans. Power Electron., vol. 23, no. 4, pp. 1759-1771, Jul. 2008.

[4] C. Wang, M. Xu, B. Lu, and F. C. Lee, "New architecture for MHz switching frequency PFC," in Proc. 22nd Annu. IEEE Appl. Power Electron. Conf. Expo., Feb. 25-Mar. 1, 2007, pp. 179-185.

[5] S. C. Tang, S. Y. R. Hui, and H. S. H. Chung, "Characterization of coreless printed circuit board (PCB) transformers," IEEE Trans. Power Electron., vol. 15, no. 6, pp. 1275-1282, Nov. 2000.

[6] S. C. Tang, S. Y. R. Hui, and H. S. H. Chung, "A low-profile low-power converter with coreless PCB isolation transformer," IEEE Trans. Power Electron., vol. 16, no. 3, pp. 311-315, May 2001.

[7] I. W. Hofsajer, J. A. Ferreira, and D. van Wyk, "Design and analysis of planar integrated L-C-T components for converters," IEEE Trans. Power Electron., vol. 15, no. 6, pp. 1221-1227, Nov. 2000.

[8] M. Ludwig, M. Duffy, T. O'Donnell, P. McCloskey, and S. C. O. Mathuna, "PCB integrated inductors for low power dc/dc converter," IEEE Trans. Power Electron., vol. 18, no. 4, pp. 937-945, Jul. 2003.

[9] R. Chen, F. Canales, B. Yang, and J. D. van Wyk, "Volumetric optima design of passive integrated power electronics module (IPEM) for distributed power system (DPS) front-end dc/dc converter," IEEE Trans. Ind. Appl., vol. 41, no. 1, pp. 9-17, Jan./Feb. 2005.

[10] E. C. W. De Jong, B. J. A. Ferreira, and P. Bauer, "Toward the next level of PCB usage in power electronic converters," IEEE Trans. Power Electron., vol. 23, no. 6, pp. 3151-3163, Nov. 2008.

[11] A. M. Niknejad and R. G. Meyer, "Analysis, design and optimization of spiral inductors and transformers for Si RF ICs," IEEE J. Solid-State Circuits, vol. 33, no. 10, pp. 1470-1481, Oct. 1998.

[12] A. Zolfaghari, A. Chan, and B. Razavi, "Stacked inductors and transformers in CMOS technology," IEEE J. Solid-State Circuit, vol. 36, no. 4, pp. 620-628, Apr. 2001.

[13] A. Massarini and M. K. Kazimierczuk, "Self-capacitance of inductors," IEEE Trans. Power Electron., vol. 12, no. 4, pp. 671-676, Jul. 1997.

[14] B. Ackermann, A. Lewalter, and E. Waffenschmidt, "Analytical modelling of winding capacitances and dielectric losses for planar transformers," in Proc. IEEE Workshop Comput. Power Electron., Aug. 15-18, 2004, pp. $2-$ 9.

[15] T. Duerbsum and G. Sauerlender, "Energy based capacitance model for magnetic devices," in Proc. 16th Annu. IEEE Appl. Power Electron. Conf. Expo., Mar. 4-8, 2001, vol. I, pp. 109-115.

[16] L. Dalessandro, F. da Silveira Cavalcante, and J. W. Kolar, "Selfcapacitance of high-voltage transformers," IEEE Trans. Power Electron., vol. 22, no. 5, pp. 2081-2092, Sep. 2007.

[17] C. K. Lee, P. C. F. Chan, and S. Y. R. Hui, "Alternating stacked inductor for mega-Hertz power converter and filtering applications," in Proc. 39th Аnnu. IEEE Power Electron. Spec. Conf., Jun. 15-19, 2008, pp. 30213027.

[18] D. Van Der Linde, C. A. M. Boon, and J. B. Klaassens, "Design of a highfrequency planar power transformer in multilayer technology," IEEE Trans. Ind. Electron., vol. 38, no. 2, pp. 135-141, Apr. 1991.

[19] Maxwell Users Guide, Ansoft Corporation, Pittsburgh, PA, Jan. 3, 1990.

[20] P. C. F. Chan, C. K. Lee, and S. Y. R. Hui, "Stray capacitance calculation of coreless planar transformers including fringing," Electron. Lett., vol. 43, no. 23 , Nov. 2007.

[21] D. K. Cheng, Field and Wave Electromagnetics, 2nd ed. Reading, MA: Addison-Wesley, Nov. 2002, pp. 72-143.

[22] W. G. Hurley and M. C. Duffy, "Calculation of self and mutual impedances in planar magnetic structures," IEEE Trans. Magn., vol. 31, no. 4, pp. 2416-2422, Jul. 1995.

[23] W. G. Hurley and M. C. Duffy, "Calculation of self- and mutual impedances in planar sandwich inductors," IEEE Trans. Magn., vol. 33, no. 3, pp. 2282-2290, May 1997.

[24] S. C. Tang, S. Y. R. Hui, and H. S. H. Chung, "Evaluation of the shielding effects on printed-circuit-board transformers using ferrite plates and copper sheets," IEEE Trans. Power Electron., vol. 17, no. 6, pp. 1080-1088, Nov. 2002. 
[25] X. Huang, K. D. T. Ngo, and G. Bloom, "Design techniques for planar windings with low resistances," in Proc. 10th Annu. IEEE Appl. Power Electron. Conf. Expo., Mar. 5-9, 1995, vol. 2, pp. 533-539.

[26] E. C. Snelling, Soft Ferrites Properties and Applications. Belfast, U.K.: Butterworth, 1998, pp. 313-335.

[27] B. R. Hunt, R. L. Lipsman, and J. Rosenberg, A Guide to MATLAB: For Beginners and Experienced Users. Cambridge, U.K.: Cambridge Univ. Press, 2006

[28] Agilent Impedance Measurement Handbook, A Guide to Measurement Technology and Techniques, 4th ed., Agilent Technologies, Santa Clara, CA, Jun. 2009.

[29] E. Waffenschmidt and B. Ackermann, "Size advantage of coreless transformers in the $\mathrm{MHz}$ range," presented at the Eur. Conf. Power Electron. Applicat. (EPE 2001), Paper DS2-9.

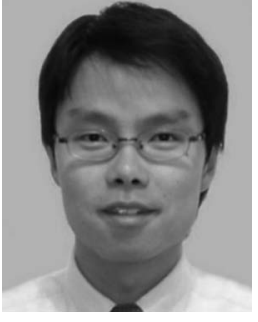

versity, Kowloon power systems, advanced inverters for renewable energy and smart grid, reactive power control for load management in renewable energy systems, contactless wireless power transmission, energy harvesting, and planar electromagnetics for high-frequency power converters.

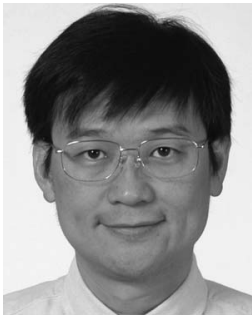

S. Y. Ron Hui (F'03) received the B.Eng. (Hons) degree from the University of Birmingham, Birmingham, U.K., in 1984, and the D.I.C. and Ph.D. degrees from the Imperial College of Science and Technology, London, U.K., in 1987.

From 1987 to 1990, he was a Lecturer at the University of Nottingham, Nottingham, U.K. In 1990, he was at the University of Technology, Sydney, Australia. In 1992, he was a Senior Lecturer at the University of Sydney, Sydney, where he became a Reader in 1995. In 1996, he joined the City University of Hong Kong (CityU), Kowloon, Hong Kong, as a Professor and was promoted to Chair Professor in 1998. From 2001 to 2004, he served as an Associate Dean of the Faculty of Science and Engineering at CityU. Since 2010, he holds the Chair Professorship at both the CityU and the Imperial College of Science and Technology, London, U.K.

He has published over 200 technical papers, including more than 140 refereed journal publications and book chapters. Over 50 of his patents have been adopted by industries.

Dr. Hui is a Fellow of the Institution of Engineering and Technology.He has been an Associate Editor (Power Conversion) of the IEEE TRANSACTIONS ON POWER ELECTRONICS since 1997, and also an Associate Editor (Lighting Technology) of the IEEE TRANSACTIONS ON INDUSTRIAL ELECTRONICS since 2007. He has been appointed twice as an IEEE Distinguished Lecturer by the IEEE Power Electronics Society in 2004 and 2006. He served as one of the 18 Administrative Committee members of the IEEE Power Electronics Society and was the Chairman of its Constitution and Bylaws Committee from 2002 to 2010. He received the Excellent Teaching Award at CityU in 1998 and the Earth Champion Award in 2008. He won an IEEE Best Paper Award from the IEEE IAS Committee on Production and Applications of Light in 2002, the IEEE Power Electronics Transactions Prize Paper Award for his publication in Wireless Battery Charging Platform Technology in 2009 and the same award in 2010 for his paper on General Photoelectrothermal theory for LED systems. His inventions on wireless charging platform technology have been adopted by the Wireless Power Consortium in the world's 1st international standard for wireless power transfer "Qi." In 2010, he was selected as the recipient of the IEEE Rudolf Chope R\&D Award by the IEEE Industrial Electronics Society and was admitted to the Fellowship of the Australian Academy of Technological Sciences and Engineering.

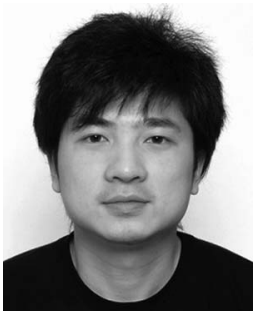

Y. P. Su (S'06) received the B.S. degree in electrical engineering from Tsinghua University, Beijing, China, in 2005, and the M.Phil. degree from City University of Hong Kong, Kowloon, Hong Kong, in 2008. He is currently working toward the Ph.D. degree from the Center for Power Electronics Systems, Virginia Polytechnic Institute and State University, Blacksburg.

From September to December 2008, he was as a Research Associate at the Center for Power Electronics, City University of Hong Kong.

His main research interests include magnetic design, circuit integration in power electronics, and low-voltage high-current dc-dc converter. 